\title{
Sistema de cartografía arqueológica predictiva: PRONOS
}

José Manuel Díaz Iglesias, José María Rodrigo Cámara, Silvia Fernández Cacho, Centro de Documentación y Estudios, IAPH

\section{ANTECEDENTES: PROYECTO MODELO ANDALUZ DE PREDICCIÓN ARQUEOLÓGICA (MAPA)}

Desde la creación del IAPH en 1989, uno de sus proyectos más relevantes ha sido la creación y acrecentamiento de un gran banco de datos del patrimonio histórico, el Sistema de Información del Patrimonio Histórico (SIPHA), de cuya gestión y mantenimiento se ha hecho cargo su Centro de Documentación y Estudios (LADRÓN DE GUEVARA SÁNCHEZ; MUÑOZ CRUZ, 2007).

La dimensión territorial de muchas de las entidades patrimoniales gestionadas por el sistema ha permitido analizar su distribución y relación con otros elementos y fenómenos del territorio en el que se insertan y con los que interactúan.

En efecto, la existencia de inventarios y registros informatizados -en este caso relacionados con el patrimonio histórico- tiene un indudable valor como repositorio de información que puede ser consultada y difundida con relativa facilidad, pero su mayor virtualidad es la posibilidad de analizarla para obtener nueva información sobre las que basar prioridades de actuación con criterios objetivables.

El IAPH, a través de su Centro de Documentación y Estudios, ha avanzado en los últimos años en el desarrollo de diversas investigaciones sobre la dimensión espacial del patrimonio histórico inmueble (FERNÁNDEZ CACHO; MONDÉJAR FERNÁNDEZ DE QUINCOCES; DÍAZ IGLESIAS, 2002; CARRERA DÍAZ; MONDÉJAR FERNÁNDEZ DE QUINCOCES; SORO CAÑAS, 2007; FERNÁNDEZ CACHO; FERNÁNDEZ SALINAS; HERNÁNDEZ LEÓN et ál., 2010). Para ello se han aplicado tecnologias de la información geográfica y métodos cuantitativos cada vez más complejos que han servido no sólo para añadir valor a la información que almacena, sino también para desarrollar métodos y técnicas que mejoren la calidad de la información difundida a través de su servicio de cartografia (FERNÁNDEZ CACHO; NAVASCUÉS FERNÁNDEZ-VITORIO; BLASCO ARANDA, 2000; DÍAZ IGLESIAS, 2007; 2010).

Éste fue el objetivo del proyecto Modelo Andaluz de Predicción Arqueológica (MAPA) que se desarrolló entre los años 2003 y 2007 (FERNÁNDEZ CACHO; RODRIGO CÁMARA, 2009). Con él se abria una linea de investigación que, con el fin de evaluar el potencial de ocupación humana del territorio en el pasado, tenía como punto de partida el estudio de la implantación y distribución te- rritorial de las evidencias arqueológicas registradas en el presente. Para ello, se realizó el análisis estadistico de las diversas relaciones detectadas entre las entidades arqueológicas y los elementos del medio biótico y abiótico, conformando el núcleo principal de las variables que, finalmente, han proporcionado un modelado regional del potencial de aparición de restos arqueológicos con representación de cinco niveles.

Tras la publicación de los resultados del proyecto MAPA en 2009, se comenzó a diseñar el Sistema de Cartografía Arqueológica Predictiva (Pronos), un sistema que automatiza el tratamiento de la cartografía y la aplicación de los procedimientos estadísticos necesarios para generar la cartografía de potencial arqueológico. En este trabajo se presenta su arquitectura básica y sus principales ámbitos de aplicación.

\section{PRONOS: DESCRIPCIÓN DE LA APLICACIÓN}

A lo largo de 2010 se ha culminado la última fase de este proyecto. De este modo, a partir de los presupuestos teóricos y metodológicos planteados, se ha diseñado y desarrollado una herramienta para la automatización de los procesos de generación y consulta de la cartografia arqueológica predictiva: Pronos. Esta aplicación se inserta conceptualmente en el ámbito de las tecnologias de la información geográfica. Con ella se dota a la Administración cultural de un innovador instrumento de gestión que permite, entre otras prestaciones, orientar las líneas de investigación futuras en materia de patrimonio arqueológico, tener accesible un documento que reforzará sus políticas preventivas y manejar una información cualificada en sus estudios de paisaje.

Para el diseño de la aplicación informática se ha partido del modelo conceptual ya definido durante la fase de investigación entre 2003 y 20071. Pronos es una aplicación para la generación automática de nuevos modelos predictivos a partir de la actualización o adición de las variables de entrada. La generación de un modelo predictivo en el contexto del MAPA es un proceso basado en un modelo matemático que tiene por objetivo analizar las relaciones entre la localización de las entidades arqueológicas conocidas (variable dependiente) y un conjunto de variables medioambientales (variables independientes) para predecir la probabilidad de presencia de yacimientos en áreas no estudiadas de la región andaluza. 

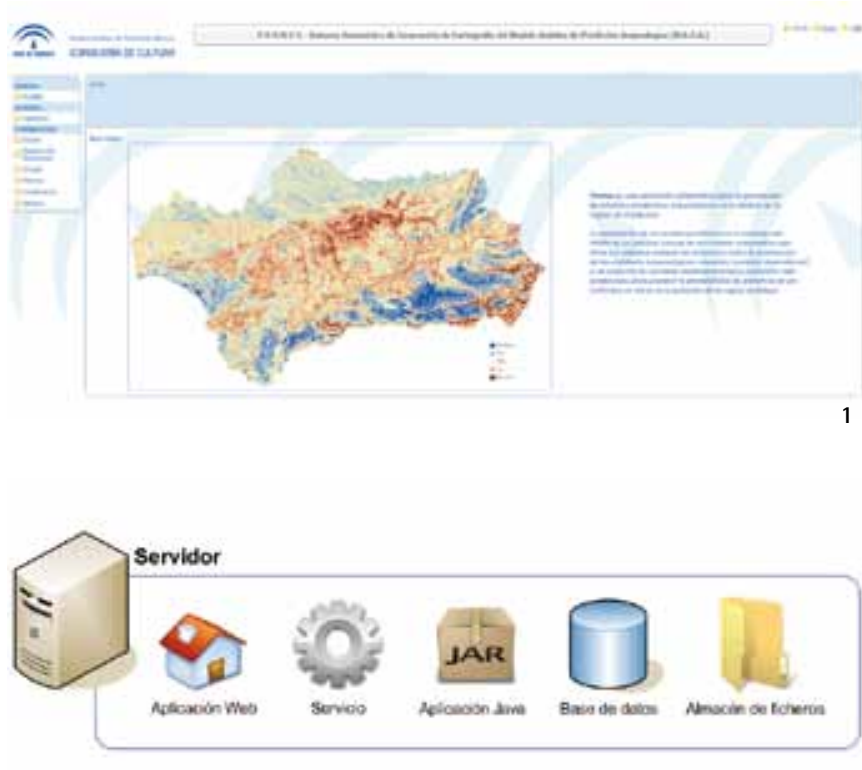

1. Interfaz de Pronos

2. Componentes de la Arquitectura de Pronos

Para ello, la aplicación Pronos realiza una serie de procesos que comprenden, en su núcleo principal, la adecuación e inclusión de la información espacial de un conjunto de variables dependientes e independientes, el cálculo y procesamiento geoestadístico de las mismas, la aplicación de un modelo matemático y la generación de resultados en formato de cartografía digital e informes de valoración estadística.

La interfaz de usuario distingue los siguientes módulos básicos:

- Consulta de los modelos generados y creación de nuevos modelos. Este módulo permite a los usuarios la consulta de los modelos creados, siendo posible examinar los parámetros de configuración del modelo y sus resultados. Por otro lado permite lanzar un nuevo modelo, seleccionando las variables que van a configurarlo.

- Gestión de las variables. Es el módulo en el que se estructura la información espacial que va a ser utilizada para la generación de nuevos modelos, agrupándose bajo diferentes pestañas. Aquí se incluyen los ficheros que contienen los recortes subregionales, los ficheros originales de las variables dependientes e independientes y los ficheros que han sido procesados a partir de los anteriores, agrupados bajo el epigrafe de variables derivadas dependientes e independientes.

- Configuración de la aplicación. Es un módulo de acceso restringido al usuario con perfil de administrador. En él se definen diferentes parámetros que incluyen la escala, el sistema de referencia, el origen de los datos, la denominación de los periodos históricos para las variables dependientes, las reclasificaciones de las variables independientes almacenadas y la gestión de usuarios.

En cuanto al modelo de despliegue o modelo físico, la infraestructura del sistema se ha concebido con una arquitectura de tipo cliente-servidor. Consta de los siguientes componentes:
- Servidor de aplicaciones. Es una aplicación web hospedada en un servidor web que sirve como interfaz para los usuarios de Pronos. Tiene acceso al almacén de ficheros, a la aplicación Java y a la base de datos.

- Bajo ella se aloja una aplicación que funciona como un servicio dentro del servidor de aplicaciones. Se encarga de almacenar los modelos procesados por la aplicación Java. El servicio de Pronos tiene acceso al almacén de ficheros y a la base de datos.

- Aplicación Java. En ella se realizan todos los cálculos necesarios para Pronos. Se encarga de procesar los modelos y de generar las variables, además de proporcionar funciones auxiliares que serán usadas por la aplicación web de Pronos. Bajo ella se han integrado las distintas librerias de algoritmos de álgebras de mapas que permiten realizar las funciones de análisis espacial y cálculos geoestadísticos. Tiene acceso al almacén de ficheros.

- Base de datos. Su finalidad es servir de almacenamiento de los datos de la aplicación. Para ello se ha optado por integrar el motor de bases de datos PostgreSOL.

- Almacenamiento de archivos: Se trata de un sistema de ficheros necesario para almacenar los archivos de gran tamaño que procesa Pronos.

Durante el proceso de diseño de la aplicación ha sido un requisito demandado para su implementación la utilización de herramientas de código abierto. En este sentido, el software necesario para el funcionamiento de Pronos, instalado en el servidor de aplicaciones, está constituido por: Servidor HTTP Apache 2, Mono, Mono Apache Server 2, Motor de Java y el Sistema Gestor de Bases de Datos Relacionales PostgreSOL.

Sin embargo, el reto fundamental ha consistido en la implementación de los operadores de álgebra de mapas necesarios para las operaciones de análisis espacial y el tratamiento de las variables. Para ello, y una vez descartadas otras opciones, se ha optado por implementar las librerias de algoritmos desarrolladas dentro del Proyecto SEXTANTE2 ${ }^{2}$ adaptando en algún caso alguna de ellas para su adecuación al tratamiento inicial que se dio en el proyecto MAPA. El conjunto de estos algoritmos puede agruparse, siguiendo la metodología clásica propuesta por Tomlin (TOMLIN, 1990), en:

- Funciones u operadores locales: La reclasificación de valores es un ejemplo de estas funciones. Está presente en los procesos de generación de todas las variables independientes, así como en los procesos de generación del modelo.

- Funciones u operadores focales o de vecindad. Este tipo de operadores está presente en los algoritmos para la generación de algunas de las variables independientes: cálculo de la altimetría relativa, de la pendiente, del aspecto, de la medida cuantitativa de la forma del terreno o de la distancia a la red hidrográfica.

Además de los algoritmos de análisis espacial se han implementado otras librerias que permiten funciones de transformación (rasterizar, recortar y unir). 
El diseño actual de Pronos ha sido concebido bajo criterios de calidad que permiten la modularidad y reutilización de los componentes. Así, por ejemplo, aunque actualmente es posible añadir variables no contempladas en el proyecto inicial, siempre que se hayan tratado y adecuado a los requisitos del sistema, la aplicación se puede ampliar sin grandes esfuerzos añadiendo, por ejemplo, otros algoritmos para el tratamiento de nuevas variables.

Por último es necesario señalar que dado que Pronos trabaja con un gran volumen de información espacial, se ha desarrollado como parte de la aplicación un sistema de gestión transparente de metadatos. Éste permite asociar a cada capa de información geográfica un fichero en formato xml con sus metadatos según el estándar ISO 19115 y su esquema de implementación definido en ISO 19139. Para ello, es un requisito general que los ficheros de información espacial que definen las variables originales de entrada lleven asociado sus correspondientes metadatos. A partir de éstos, la aplicación genera un fichero de metadatos básicos, siguiendo los mismos estándares, tanto para las variables derivadas generadas como para las capas de información que componen un modelo.

\section{MARCOS DE APLICACIÓN DE PRONOS}

Con esta aplicación el IAPH ha desarrollado una herramienta que permite sin grandes esfuerzos la generación y actualización de la cartografía de potencial arqueológico de Andalucia. Aunque esta información pueda ser de utilidad en diferentes contextos, en principio, su uso se centra prioritariamente tanto en el ámbito de la gestión e investigación patrimonial como en el de la planificación territorial.

En cuanto al primero, en el contexto de los proyectos de investigación que desarrolla el IAPH, esta información cualificada puede ser utilizada en los estudios de paisaje cultural, así como en diferentes líneas de trabajo relacionadas con tareas de identificación y definición de zonas de alto potencial arqueológico. Además de servir de apoyo a proyectos específicos, Pronos facilita la posibilidad de seguir avanzando en la definición del modelo predictivo, mediante la actualización de la información espacial de entrada o mediante el ensayo y combinación de nuevas variables.

Por otro lado, con respecto a la difusión de esta información, los resultados generados van a formar parte del servicio de cartografía de esta institución. De este modo, además de la información de las entidades patrimoniales, este servicio va a ofrecer esta cartografia en las solicitudes de información patrimonial de proyectos que, por ejemplo, incluyan en su tramitación una evaluación de impacto ambiental. Dado que en estos documentos se trata de realizar un análisis predictivo para valorar y prevenir los efectos de una actuación sobre el medio ambiente, la incorporación de esta información puede aportar un valor cualitativo.
En el contexto de la gestión cultural, Pronos proporciona documentos que combinados con el conocimiento actual de las entidades arqueológicas pueden servir de apoyo para la toma de decisiones en las políticas culturales, delimitando, por ejemplo, áreas de investigación o intervención prioritaria definidas por un escaso conocimiento y un alto potencial de ocupación.

Por último, en el ámbito de la planificación territorial, la cartografía generada puede ser un instrumento para tener en consideración en la redacción del planeamiento territorial regional y subregional. En este sentido, la incorporación de esta información puede servir como apoyo a la definición de áreas que por su elevado potencial arqueológico puedan someterse a determinadas cautelas. Es, por ello, una herramienta que podrá apoyar en el futuro el desarrollo una gestión arqueológica basada en la prevención y planificación de actividades que eviten o, al menos, minimicen afecciones y riesgos.

\section{Notas}

${ }^{1}$ Véase los capítulos 3 y 4 de la publicación electrónica MAPA.

2 SEXTANTE es un proyecto desarrollado por la Universidad de Extremadura para la Junta de Extremadura. Para más información puede consultarse en http://forge. osor.eu/plugins/wiki/index.php?P\%C3\%A1 gina\%20principal\&tid=13\&type =g

\section{Bibliografía}

CARRERA DÍAZ, G.; MONDÉJAR FERNÁNDEZ DE QUINCOCES, P.; SORO CAÑAS, S. (2007) La información del Patrimonio Inmueble. En LADRÓN DE GUEVARA SÁNCHEZ, M. C.; MUÑOZ CRUZ, V. (coord.) El Sistema de Información del Patrimonio Histórico de Andalucía. PH Cuadernos, n. ${ }^{\circ}$ XX. Sevilla: Instituto Andaluz del Patrimonio Histórico, Consejeria de Cultura, 2007, pp. 174-189 DÍAZ IGLESIAS, J. M. (2007) El subsistema de información geográfica. En LADRÓN DE GUEVARA SÁNCHEZ, M.C.; MUÑOZ CRUZ, V. (coord.) El Sistema de Información del Patrimonio Histórico de Andalucia. PH Cuadernos, n. ${ }^{\circ}$ XX. Sevilla: Instituto Andaluz del Patrimonio Histórico, Consejería de Cultura, 2007, pp. 160-170

DíAZ IGLESIAS, J. M. (2010) El Servicio de Cartografía Digital del Instituto Andaluz del Patrimonio Histórico. PH Boletín del IAPH, n. ${ }^{7} 73,2010$, pp. 32-41 FERNÁNDEZ CACHO, S.; NAVASCUÉS FERNÁNDEZ-VITORIO, R.; BLASCO ARANDA, E. M. (2000) GeoARQUEOS: A System for the creation, updating and validation of the digital cartography of the Andalusian Archaeological Heritage. Archeologia e Calcolatori, n.011, 2000, pp. 359-373 FERNÁNDEZ CACHO, S.; RODRIGO CÁMARA, J. M. (2009) (coord.) MAPA: Modelo Andaluz de Predicción Arqueológica [en linea]. e-ph Cuadernos, n. ${ }^{\circ}$ 1, 2009 < http://www.juntadeandalucia.es/cultura/iaph/nav/ modeloandaluzdeprediccionarqueologica> [consulta: 07/12/10]

FERNÁNDEZ CACHO, S.; MONDÉJAR FERNÁNDEZ DE QUINCOCES, P.; DÍAZ IGLESIAS, J. M. (2002) La información de Patrimonio Arqueológico de Andalucia: Valoración general. En FERNÁNDEZ CACHO, S. (ed.) ARQUEOS. Sistema de Información del Patrimonio Arqueológico de Andalucía. Granada: Instituto Andaluz del Patrimonio Histórico, Consejería de Cultura, 2002, pp. 143-166 FERNÁNDEZ CACHO, S.; FERNÁNDEZ SALINAS, V.; HERNÁNDEZ LEÓN, E. et ál. (2010) Paisaje y Patrimonio Cultural en Andalucía. Tiempo, Usos e Imágenes. Colección PH Cuadernos, n. ${ }^{\circ}$ 27, 2v. Sevilla: Instituto Andaluz del Patrimonio Histórico, Consejería de Cultura, 2010

LADRÓN DE GUEVARA SÁNCHEZ, M. C.; MUÑOZ CRUZ, V. (2007) (coord.) El Sistema de Información del Patrimonio Histórico de Andalucía. PH Cuadernos, n. ${ }^{\circ}$ XX. Sevilla: Instituto Andaluz del Patrimonio Histórico, Consejería de Cultura, 2007 TOMLIN, C. D. (1990) Geographic information systems and catographic modeling. New Jersey: Englewood Cliffs-Prentice Hall, 1990 